\title{
Do Tangible User Interfaces Impact Spatial Cognition in Collaborative Design?
}

\author{
MARY LOU MAHER AND MI JEONG KIM \\ Key Centre of Design Computing and Cognition \\ University of Sydney, Australia 2006 \\ Emailaddress: mary@arch.usyd.edu.au/mkim9133@arch.usyd.edu.au
}

\begin{abstract}
Developments in digital design workbenches that combine Augmented Reality (AR) systems and tangible user interfaces (TUIs) on a horizontal display surface provide a new kind of physical and digital environment for collaborative design. The combination of tangible interaction with AR display techniques change the dynamics of the collaboration and have an impact on the designers' perception of 3D models. We are studying the effects of TUIs on designers' spatial cognition and design communication in order to identify how such tangible systems can be used to provide better support for collaborative design. Specifically, we compared tangible user interfaces (TUIs) with graphical user interfaces (GUIs) in a collaborative design task with a focus on characterising the impact these user interfaces have on spatial cognition.
\end{abstract}

\section{INTRODUCTION}

Digital design workbenches, table-top Augmented Reality (AR) systems equipped with tangible user interfaces (TUIs), have been proposed as an alternative for the design review meetings since they allow designers to intuitively modify the spatial qualities of 3D designs and keep communication channels open by preserving traditional mechanisms such as verbal and non-verbal communication. This research focuses on the way the digital design workbench supports design thinking and design communication. Several researchers have proposed that such tangible interaction combined with AR display techniques might affect designers' cognition and communication (Tang, 1991; Bekker et al., 1995). However, they have not posed any evidence in a systematic way. To date, the central preoccupation of research on TUIs has been in a developmental direction, which usually describes the fundamental ideas behind their tangible systems, their prototype implementation, possible application areas and some initial usability results. We aim to obtain empirical evidence about the potential impact of TUIs by investigating if and how the tangible presence of the virtual objects on the design workbench affects designers' spatial cognition and design communication in collaborative design. This paper presents some preliminary results of a pilot study using protocol analysis, which considers what factors are associated with designers' spatial cognition in 3D modelling.

\section{TANGIBLE PRESENCE AND SPATIAL COGNITION}

Since a human's cognitive ability is strongly bounded, to carry out complex reasoning without the aid of tools is very difficult. Norman (1991) defined tools as cognitive artefacts, which do not modify the computational power of the human's cognitive ability but instead modify the content of the knowledge involved in the elaboration process (Rizzo et al., 1995). That is, tools of thought - cognitive artefacts - are external aids that enhance cognitive abilities. Thus, we predict that designers' perception of spatial knowledge will be improved when using TUIs, and this may be due to the tangible presence of virtual objects.

\subsection{Tangible user interfaces and physical interaction}

AR technology blends reality and virtuality to allow the seamless interaction between physical and digital worlds. Thus AR research has focused on the linkage between digital information and physical objects by superimposing, tagging and tracking objects. The term "augmented reality" is often used to refer to such TUIs in which 2D and 3D computer graphics are superimposed on physical objects. TUIs turn the physical 
objects into input and output devices for computer interfaces to restore the richness of the physical world in human-computer interaction. Arias et al. (1997) argued that new HCI approaches need to combine physical and digital environments to augment the weaknesses of the other by using the strengths of each since the "reflective conversations" are very different between the two environments. Digital design workbenches that combine AR technologies and TUIs provide a physical interaction by putting tangible 3D objects containing digital information into a single environment.

According to Wang et al. (2001), the strengths of physical interaction can be explained by two aspects. Firstly, physical interaction provides direct, naïve manipulability and intuitive understanding. It is very natural to pick up and place a physical object; certain characteristics such as size, weight, colour and shape can be used to communicate meaning. Secondly, physical interaction provides tactile interaction; the sense of touch provides an additional dimension of interaction. Seichter and Kvan (2004) posed the concept of "augmented affordance" to explain interactions using TUIs in AR systems. Norman (1988) regarded the study of affordances of objects, originated by Gibson (1979), as the start of a psychology of things since affordances result from the mental interpretation of things, based on our past knowledge and experience applied to our perception of the things. He proposed that "affordance" refers to the perceived and actual properties of the thing that determine just how the thing could possibly be used. According to Seichter and Kvan (2004), TUIs can be seen as offering a conduit between the real or perceived affordances implied by the physical properties of the interface tool and the affordances created by the digital behaviours in the virtualised interface.

\subsection{Designers' spatial cognition and spatial representation}

As a consequence of the diversity of approaches and related disciplines, there is little consistency in what is meant by the term "spatial" (Foreman \& Gillett, 1997). We associate the designers' perception of the form and spatial relationships of the design components with the designers' spatial cognition. The meaning of 'space' to the designers is not an abstract of empty space, but rather of the identity and the relative locations of the objects in space. Space then is decomposed into particular objects and the spatial relationships among them viewed from a particular perspective. The spatial relationships may include functional reasoning since design is required to satisfy intended functions. In addition, it has been argued that touch is also a spatial modality, where the close linkage between motor and spatial processes has been emphasized. Kinaesthetic information through a haptic system provides us with the ability to construct a spatial map of objects that we touch (Loomis \& Lederman, 1986). It is the movement of a hand repeatedly colliding with objects that comes to define extra-personal space for each individual, as a consequence of repeatedly experienced associations (Foreman \& Gillett, 1997). Thus, the movement simulated by the mouse in desk-top systems lacks tactile and kinaesthetic feedback that normally accompanies movement.

Based on the assumption that people often use general purpose verbs and prepositions when the context is sufficiently clear to disambiguate them, we will investigate language spatial representation. Language draws on spatial cognition so that we can talk about what we perceive and it thereby provides a window on the nature of spatial cognition (Anibaldi \& Nualláin, 1998). Jackendoff (1983) suggested a level of mental representation devoted to encoding the geometric properties of objects and the relationships among them in space, in order to express our spatial experience in talking about objects and talking about spatial relationships. Gesture is also recognized as a good vehicle for capturing visual and spatial information as it is associated with visuospatial content (Wagner et al., 2004). People produce some gestures along with their speech, and such speech-accompanying gestures are not just hand moving (Lavergne \& Kimura, 1987). Speech and gesture are both characterizing the spatial relationships among entities, which are closely related to and may even be beneficial for cognitive processing (Goldin-Meadow, 2003). Wagner et al. (2004) found that the movement of hands facilitates recall of visuospatial items as well as verbal items.

\subsection{Digital Design Workbenches}

We reviewed various digital design workbenches: metaDESK, iNavigator, BUILD-IT, PSyBench, URP, MIXdesign and ARTHUR system. Ulmer and Ishii (1997) constructed the metaDESK system with a focus on physical interaction to manipulate the digital environment. Standard 2D GUI elements like windows, icons, and menus, are given a physical instantiation as wooden frames, 'phicons, and trays, respectively. iNavigator is a CAD platform for designers to navigate and construct $3 \mathrm{D}$ models, which consists of a vertical tablet device for displaying a dynamic building section view and a horizontal table surface for 
displaying the corresponding building plan geometry. From the user's perspective, the display tablet is served as "a cutting plane" (Lee et al., 2003). BUILD-IT developed by Fjeld et al. (1998) is a cooperative planning tool consisting of a table, bricks and a screen, which allows a group of designers, co-located around the table, to interact, by means of physical bricks, with models in a virtual 3D setting. A plan view of the scene is projected onto the table and a perspective view of the scene is projected on the wall. Brave et al. (1998) designed PSyBench and inTouch, employing telemanipulation technology to create the illusion of shared physical objects that distant users are interacting with. Although still in the early stage, it shows the potential of distributed tangible interfaces. URP developed by MIT media lab is a luminous tangible workbench for urban planning that integrates functions addressing a broad range of the field's concerns such as cast shadows, reflections and windflow into a single, physically based workbench setting. The URP system uses pre-existing building models as input to an urban planning system (Underkoffler \& Ishii, 1999). MIXDesign allows architects to interact with a real scale model of the design by using a paddle in a normal working setting, and also presents an enhanced version of the scale model with 3D virtual objects registered to the real ones (Dias et al., 2002). ARTHUR system is an Augmented Round Table for architecture and urban planning, where virtual 3D objects are projected into the common working environment by semi-transparent stereoscopic head mounted display (HMDs). Placeholder objects (PHOs) and wand are used to control virtual objects (Granum et al., 2003).

These various configurations of digital workbenches, with and without augmented reality, show a trend in developing technology that supports designers in creating and interacting with digital models that go beyond the traditional human-computer interface of the keyboard, mouse, and vertical screen. The different configurations described above draw on specific intended uses to define the components and their configuration. Few of the publications about digital workbenches evaluate the new interface technology with respect to spatial cognition or improved understanding of the spatial relationships of the components of the digital model. In this paper, we consider the existing digital workbenches as defining a class of design environments that use TUIs to be a departure from the traditional GUIs that designers are currently using to create and interact with a digital design model. While TUIs and GUIs will continue to be alternative design environments for digital models, we focus on the differences between them in order to clarify the role and benefit of TUIs for designers.

\section{COMPARING GUI-BASED WITH TUI-BASED COLLABORATION}

In devising an experiment that can highlight the expected improvement in spatial cognition while using TUIs, various scenarios have been considered: Face-to-face collaboration with physical models versus TUI-based collaboration with tangible digital models; Face-to-face collaboration with pen and paper versus GUI-based collaboration with mouse and keyboard; GUI-based collaboration with intangible digital models versus TUI-based collaboration with tangible digital models. We chose the third category for this research because it will enable us to verify if and in what way tangible interaction affect designers' spatial understanding of 3D models in computer-mediated collaborative design.

\subsection{Design collaboration in GUI vs. TUI environments}

A GUI is a graphical user interface to a computer, which features a pointing device, icons and menus, and desktop on the display screen. Thus, we consider a 3D CAD system for the design environment in a GUI environment. Current CAD systems allow designers to explore design alternatives as modifiable models, but they produce indirect interaction with 3D models using a mouse. Despite the physical form, the mouse has no physical contextual awareness and lacks the efficiency of specialized tools. It is regarded as a timemultiplexed input device controlling different functions at different times (Fitzmaurice, 1996). The ability to use a single device for several tasks is a major benefit of the GUI, but given the nature of interaction where only one person can edit the model at a time, the GUI environment may change interactivity in collaborative design (Magerkurth, 2002). On the other hand, TUIs have been offering an alternative to standard computer user interfaces, where physical objects are representations and controls for digital information, thereby restoring some of the tangibility of physical models. We consider a digital design workbench for the design configuration in a TUI environment. The digital design workbench is specifically configured for 3D design and visualization (Daruwala, 2004), where designers can manipulate $3 \mathrm{D}$ virtual objects in a semi-immersive environment in real time and can be spatially aware of each other as well as the design. The direct hands-on style of interaction afforded by the physical objects provides 
designers concurrent access to multiple, specialized input devices. That is, they are "space-multiplexed" input devices, which can be attached to different functions, each independently accessible (Fitzmaurice, 1996). Using AR techniques, TUIs merge the display and task space, which may support shared understanding due to the interaction that draws out ideas in a conversational manner (Arias et al., 2000).

\subsection{Experiment set-up}

We chose ArchiCAD as an application for the GUI-based collaboration and a design workbench constructed by Daruwala and Maher (2004) for the TUI-based collaboration. Inspired by the fact that tabletops and walls are the main working surfaces during collaborative design sessions (Ma et al., 2003), they proposed a customized digital workbench that provides both horizontal and vertical display surfaces to facilitate multiple views of the 3D model. In addition they included a collection of 3D blocks with tracking markers in ARToolKit to offer a form of tactile influence on the design as handles to the virtual objects. The digital workbench environment is designed to demonstrate a range of novel input techniques for 3D design, so it is not engineered as a targeted end-user application.

Table 1 Two experiment settings

\begin{tabular}{l||l|l}
\hline \multicolumn{2}{l|}{ GUI-based collaboration } & TUI-based Collaboration \\
\hline Hardware & Desktop computer - Mouse \& Keyboard & Digital design workbench - 3D blocks \\
\hline Application & ArchiCAD & ARToolkit \\
\hline Display/Task space & Vertical LCD screen/a mouse \& keyboard & Vertical LCD screen \& Horizontal table/Horizontal table \\
\hline & &
\end{tabular}

To simulate a situation similar to a design review meeting where designers review the current state of the 3D plan and modify it, we developed two renovation scenarios of the same degree of difficulty: the layout of a home office apartment and the layout of an interior design office. Designers are required to collaborate on renovating the studio to satisfy intended functions to a pre-defined set of specifications in design briefs. The designers did not have access to a pen device or to the 2D view in ArchiCAD to make the conditions similar to the TUI-based collaboration. A set of 3D objects were made available in the application's library in order for the designers to focus on design configuration rather than designing the furniture components during the design sessions. The working time in each design environment was limited to 20 minutes.

Table 2 Experiment design

\begin{tabular}{|c|c|c|c|c|c|c|}
\hline & \multicolumn{2}{|c|}{ Experiment 1} & \multicolumn{2}{|c|}{ Experiment 2} & \multicolumn{2}{|c|}{ Experiment 3} \\
\hline Sessions/Task & $1^{\text {st }}$ GUI (TA) & $2^{\text {nd }}$ TUI (TB) & $1^{\text {st }}$ TUI (TA) & $2^{\text {nd }}$ GUI (TB) & $1^{\text {st }}$ TUI (TB) & $2^{\text {nd }}$ GUI (TA) \\
\hline Activity & \multicolumn{6}{|c|}{ Extending and renovating the studio by placing the furniture } \\
\hline Subjects & \multicolumn{6}{|c|}{ A pair of $2^{\text {nd }}$ year architecture students } \\
\hline 2D tool/View & \multicolumn{6}{|c|}{ No pen was allowed to use / 3D view in ArchiCAD was required } \\
\hline library & \multicolumn{6}{|c|}{30 pieces of furniture were provided in order for designers to focus on design rather than furniture selection } \\
\hline
\end{tabular}

\section{SEGMENTATION AND COGNITIVE ACTIONS}

We have done a pilot study that is an adaptation of protocol analysis, a methodology for studying problem solving processes that has been widely used in design cognition. Rather than ask the designers to think aloud, we recorded their conversation and gestures while they were collaborating on a predefined design task. The data collected for analysis includes verbal description of spatial knowledge and non-verbal data such as gestures. We are not trying to capture evidence of all of the designers' cognitive actions during the design process, but instead focus on the contents of what designers do, attend to, and say while designing, looking for their perception of discovering new spatial information and actions that create new functions in the design.

\subsection{Segmentation}

Segmentation is dividing the protocol into small units according to a rule. One way of segmentation is to divide protocols based on verbalization events such as pauses or syntactic markers for complete phrases 
and sentences (Ericsson and Simon, 1993). Another way is looking at the content of the protocol, and divide the protocols into small units along lines of designer's intentions (Suwa et al., 1998). We take the latter approach, thus a change in subjects' intention or the contents of their actions flagged the start of a new segment. Focusing on a specific part of a design, designers emphasized a different aspect of the design of that specific part, which showed what they actually were doing or attending to. Consequently, a single segment sometimes comprises one sentence, and sometimes several sentences.

\begin{tabular}{c||c|l|c}
\multicolumn{2}{l}{ Table 3 Segments of the protocols from one of the TUI sessions } \\
\hline Seg. $\mathbf{~}$ & Time & \multicolumn{1}{c|}{ Transcript } & \multicolumn{1}{c}{ Intentions } \\
\hline 9 & $1: 40$ & $\begin{array}{l}\text { S: Cos I've seen in how in some studio apartments how they've got these } \\
\text { bookshelves that partition the room ....and then just chuck some crap in there }\end{array}$ & $\begin{array}{l}\text { S is retrieving previous knowledge on } \\
\text { bookshelves that partition the room. }\end{array}$ \\
\hline 10 & $1: 49$ & $\begin{array}{l}\text { A: Okay. Um. Should we go about rearranging it? } \\
\text { S: Alright, so...we're gonna move these, like, that way } \\
\text { A: So, first, these are all living stuff but you're uh... }\end{array}$ & $\begin{array}{l}\text { They are rearranging 3D blocks by moving } \\
\text { all living stuff to other side. }\end{array}$ \\
\hline 11 & $2: 04$ & $\begin{array}{l}\text { S: How about if we partition in there and we... } \\
\text { A: ...Put some bookshelves like this. Uhh.. yeah... some bookshelves }\end{array}$ & $\begin{array}{l}\text { A is putting bookshelves on the horizontal } \\
\text { surface attending to partition }\end{array}$ \\
\hline
\end{tabular}

\subsection{Cognitive actions}

For each segment, we classified designers' cognitive actions into four categories including visual and nonvisual information based on Suwa's definition (1998): 3D modelling actions, perceptual actions, functional actions and design communication. The three categories correspond to the levels at which incoming information is thought to be processed in human cognition. 3D modelling actions correspond to sensory level, perceptual actions to perceptual, and functional actions to semantic. Another action category, the design communication, is included to reveal specific aspects of the collaborative interactions. We paid attention to the information of whether or not actions are new for each design action and the dependency on each other in order to measure the correlation between them and designers' spatial cognition. The first category, 3D modelling actions, refers to physical actions that are functionally processed with design thinking. This category includes the selection, placement and relocation of pieces of 3D blocks made by designers. The second category, perceptual actions, refers to actions of attending to visuo-spatial features of the artefact or spaces they are designing with. Perceptual actions are inherently dependent on physical actions. The attention to the feature or relations, creation of new space or relations, and unexpected discovery of objects or spaces that occurred were investigated as a measure of designers' perceptive abilities for spatial knowledge. The third category, functional actions, refers to actions of conceiving of non-visual information, but something with which the designers associate visual information. Whenever instances of functional actions were found it was necessary to interpret what perceptual actions or 3D modelling actions were dependent on them. In terms of functional actions, we focused on discovering 'Reinterpretation', which was applied to a segment when a designer defined a different function from previous one when s/he revisited. The fourth category, design communications, refers to actions of communicating with one another regarding design ideas and the completion of the design task itself.

\section{ANALYSIS}

The following analysis is a preliminary interpretation of the data collected. We focussed on finding patterns of designers' behaviours and cognitive actions by interpreting the information shifts, specifically looking for significant differences in the data collected from the GUI sessions and the data collected in the TUI sessions.

\subsection{Observation of designers' behaviours}

Through the observation, we noticed that designers in the GUI sessions discussed ideas verbally and decided on a solution before performing 3D modelling actions whereas designers in the TUI sessions communicated design ideas by gesturing at and moving the objects visually and decided on the location of each piece of furniture as they were manipulating 3D blocks. That is, designers in the GUI sessions spent a lot of time explaining their ideas to one another verbally while designers in the TUI sessions used 3D blocks to test and visualize design ideas. In terms of collaborative interactions, the TUI environment enabled designers to collaborate on handling the 3D blocks more interactively by allowing concurrent access to the $3 \mathrm{D}$ blocks and to produce more revisited $3 \mathrm{D}$ modelling actions before producing the final outputs. These results may be caused by the different properties of the tools. Designers in the GUI environment shared a single mouse compared to multiple 3D blocks, thus one designer mainly 
manipulated the mouse and the other explained to his partner what they were focusing on. On the other hand, with the direct, naïve manipulability of physical objects and rapid visualization, designers in the TUI environment seemed to produce more multiple cognitive actions and completed the design tasks faster.

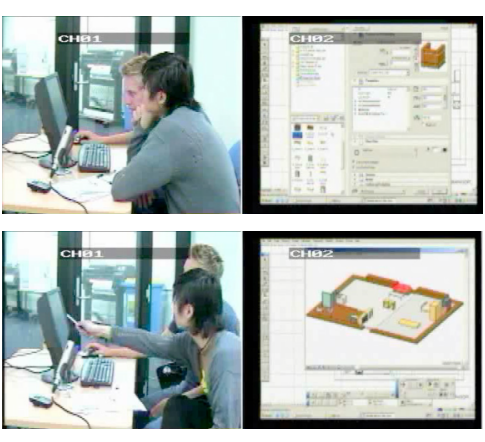

Figure 1 GUI-based collaboration
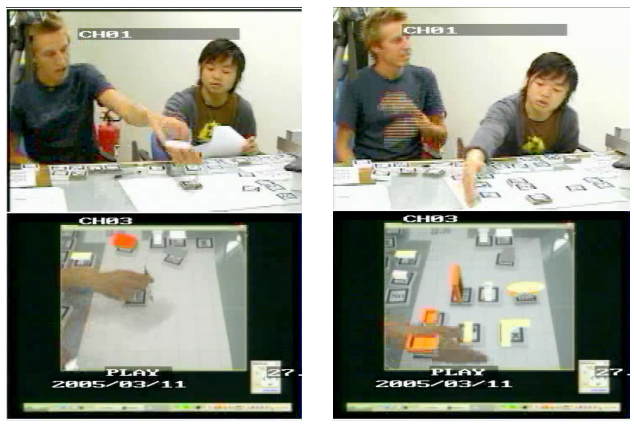

Figure 2 TUI-based collaboration

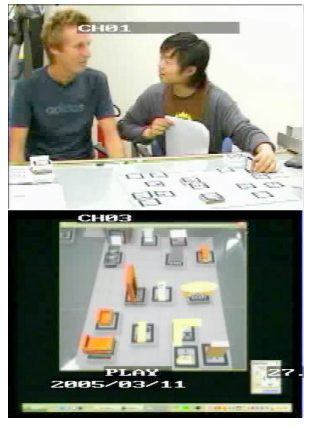

The above results motivated us to be interested in the revisited $3 \mathrm{D}$ modelling actions in terms of idea developments and designers' spatial cognition. We speculate that the revisited 3D modelling actions in the TUI sessions uncover information that is hidden or hard to compute mentally and suggest that this will play an important role in supporting designers' spatial cognition and producing a design solution.

\subsection{Cognitive actions in the GUI and TUI sessions}

Looking more in depth into the content of cognitive actions, we found different patterns of behaviour between the GUI and TUI sessions. In terms of perceiving the location of an object or space, designers in the GUI environment focused on the individual location itself while designers in the TUI environment attended more to spatial relations among objects or spaces. For example, when designers worked on the layout of a sink in the home office apartment, designers in the GUI session just clarified the location of the sink without noticing the problem in relation to the bedroom whereas designers in the TUI sessions found that the current location of the sink is wrong by perceiving the spatial relation with the bedroom.

Table 4 Perceptual actions on the location of a sink

\begin{tabular}{|c|c|c|c|}
\hline Session & $\begin{array}{c}\text { Transcript (GUI) } \\
\end{array}$ & Session & Transcript (TUI) \\
\hline \multirow{2}{*}{ GUI 2} & \multirow{2}{*}{$\begin{array}{l}\text { B: Kitchen and dining area } \\
\text { A: Yep } \\
\text { B: Which she does not yet have... well she has a sink } \\
\text { [laugh] in her ba-bedroom, and then living/meeting } \\
\text { area } \\
\text { A: Yep... and a working area }\end{array}$} & TUI 1 & $\begin{array}{l}\text { A: It shouldn't be near the bathroom or I mean, I think it shouldn't be } \\
\text { near the bedroom, sorry. It shouldn't have a kitchen sink. } \\
\text { S: Yeah that's what I was thinking. Why is it next to the bed? } \\
\text { A: It's a bit odd, and it's also just....not...normal }\end{array}$ \\
\hline & & TUI 3 & $\begin{array}{l}\text { B: coz.. The sink sink, sink dosen't need to be in the bedroom } \\
\text { A: needs to be in the kitchen } \\
\text { B: yeah sink in the kitchen. sink over here for now } \\
\text { A: } \mathrm{mm} \mathrm{hmm}\end{array}$ \\
\hline
\end{tabular}

When placing an object in the GUI environment, designers put it in a relevant area simply considering the function of the object, and sometimes they attended to the shape or style of the object itself. On the other hand, designers in the TUI environment created and attended to a new spatial relation created by placing an object, with respect to other objects or space around it. For example, regarding the placement of a new desk, designers in the GUI session emphasized the function of a desk for a computer programmer and placed it in the corner. However, designers in a TUI session considered two locations for the desk, in the corner or near the window, and then they decided to put it near the window because the designer can look out the window by creating a spatial relation between the desk and window.

Table 5 Cognitive actions on the placement of a desk

\begin{tabular}{|c|c|c|c|}
\hline Session & Transcript (GUI) & Session & Transcript (TUI) \\
\hline GUI 2 & $\begin{array}{l}\text { B: we need a work table A: desk and stuff } \\
\text { B: she's a prgrammer right? so she'd need a, ummm } \\
\text { A: that one's got a little computer thing on it } \\
\text { B: hummm (as in yeah) A: that one? } \\
\begin{array}{ll}\text { B: yeah } \quad \text { A: and that can go in the corner.... }\end{array}\end{array}$ & TUI 3 & $\begin{array}{l}\text { B: we need a desk, first of all, for his um office area.. maybe one } \\
\text { of this..is it like a desk? }\end{array}$ \\
\hline
\end{tabular}

In comparison to the GUI system, the TUI system allowed designers to discover a space and features of an existing object unexpectedly when it is revisited, and which were never paid attention to. These perceptual actions were classified as unexpected discoveries, which are usually associated with 'Re-interpretation' 
actions. Re-interpretation and unexpected discoveries have been regarded as the driving force for exploration of new design ideas, thus we can hypothesize that the TUI system facilitates generating ideas by improving designers' spatial cognition. However, it is not yet clear that the idea fluency is directly connected to the quality of the final output. The following are examples of unexpected discoveries and reinterpretation extracted from the verbal protocols of the TUI sessions.

Table 6 Unexpected discoveries and Re-interpretation in the TUI sessions

\begin{tabular}{|c|c|}
\hline Transcript & Interpretation \\
\hline $\begin{array}{l}\text { B: Just little bit layout is not very.... } \\
\text { A: You don't like how they have drawers in the middle? } \\
\text { A: You end up with empty space in the middle. how this sofa faces onto her }\end{array}$ & $\begin{array}{l}\text { B felt that the layout is little bit strange and then } \mathrm{A} \text { is discovering } \\
\text { an empty space in the middle. } \\
\text { (Unexpected discovery) }\end{array}$ \\
\hline $\begin{array}{l}\text { B: and ahh... the dining table can go... can go her... near the kitchen } \\
\text { A: I'm thinking of having the dining.. The kitchen and dining area closer to.. } \\
\text { B: Closer to the bed? } \\
\text { A: Yeah. pit it closer to this section here } \\
\text { B: That ahh That might get crowded over there. I could...move it around here? }\end{array}$ & $\begin{array}{l}\mathrm{B} \text { is discovering that the area is getting crowded (feature) by } \\
\text { placing the dining table and sink near the bed } \\
\text { (Unexpected discovery) }\end{array}$ \\
\hline $\begin{array}{l}\text { S: (Interrupt) ya know how they have those kitchens that are just two long rows? } \\
\text { A: Oh yeh } \\
\text { S: And then that would be like, become like the bar. The breakfast bar. }\end{array}$ & $\begin{array}{l}\text { After discovered the shape of kitchen to be two long rows } \\
\text { (Unexpected discovery), A is defining a new function of the } \\
\text { cabinet in the kitchen. (Re-interpretation) }\end{array}$ \\
\hline
\end{tabular}

\section{CONCLUSION}

The results of the pilot study indicated that the GUI and TUI sessions produce different outcomes in terms of designers' cognitive actions. Compared to the GUI sessions, designers in the TUI sessions exhibited the following patterns of behaviour:

- performed multiple cognitive actions in a shorter time

- re-visited a previous design frequently while coordinating design ideas

- created and attended to spatial relations such as local and global relations

- discovered a space or feature of an existing object unexpectedly

- produced more re-interpretation actions

From the above results, we can assume that the TUI system is an effective design environment for the spatial layout design since it encourages designers to attend to or create spatial relations between artefacts or spaces. However, more protocols have to be analysed to reinforce these findings.

In the next phases, we will consider different experimental situations including a single designer using the think aloud method, we will develop a coding scheme derived from our observations in the pilot study and theories of spatial cognition, and will analyse the results using both qualitative and quantitative methods.

While there may be significant differences in spatial cognition in using TUIs, we do not think that digital workbenches will replace GUI systems. Rather, new developments in TUIs will provide alternative design environments that complement existing GUI systems. Knowledge of the implications of the differences in spatial cognition provide a basis for developing and implementing new design environments as well as provide guidelines for their most effective use.

\section{REFERENCES}

Anibaldi, L. and Nualláin, S. (1998) A Computational multi-layered model for the interpretation of locative expressions. In Nualláin, S. (ed.) Spatial Cognition. Jonh Benjamins B.V. pp249-266.

Arias, E. G., Eden, H. and Fischer, G. (1997) Enhancing communication, facilitating shared understanding, and creating better artefacts by integrating physical and computational media for design. In Proceedings of the Conference on Designing Interactive Systems, ACM Press, New York, NY, 1-12.

Arias, E., Eden, H., Fischer, G., Gorman, A. and Scharff, E. (2000) Transcending the individual human mind - create shared understanding through collaborative design. ACM Transactions on Computer Human Interaction, 7 (1), pp 84-113.

ARToolKi. http://www.hitl.washington.edu/artoolkit/tutorials.htm

Bekker, M.M., Olson, J.S. and Olson, G.M. (1995) Analysis of gestures in face-to-face design teams provides guidance for how to use groupware in design. In Proceedings of the Symposium on Designing Interactive Systems (DIS)'95. pp 157-166. 
Brave, S., Ishii, H. and Dahley, A. (1998) Tangible interface for remote collaboration and communication. In Proceedings of CHI'99. pp 394-401.

Daruwala, Y. (2004) 3DT: Tangible input techniques used for 3D design \& visualization. Honors thesis, the University of Sydney.

Dias, J.M.S., Santos, P. and Diniz, N. (2002) Tangible interaction for conceptual architectural design. In Workshop of the First IEEE International Augmented Reality Toolkit 2002.

Ericsson, K. A. and Simon, H. A. (1993) A protocol analysis: verbal reports as data (revised edn). MIT Press, Cambridge MA

Fitzmaurice, G. (1996) Graspable User Interfaces. PhD thesis, the University of Toronto.

Fjeld, M., Bichsel, M. and Rauterberg, M. (1998) BUILD-IT: an intuitive design tool based on direct object manipulation. In Gesture and Sign Language in Human-Computer Interaction, Lecture Notes in Artificial Intelligence, Vol. 1371, Wachsmut and Fröhlich, Editors, Springer-Verlag, Berlin. pp 297-308.

Foreman, N. and Gillett, R. (Eds.) (1997) Handbook of spatial research paradigms and methodologies Vol. a. Hove, UK: Psychology Press.

Gibson (1979) The ecological approach to visual perception. Erlbaum Associates, New York.

Goldin-Meadow, S. (2003) Hearing gesture: How our hands help us think. Cambridge, MA: Harvard University Press.

Granum, E., Moeslund, T.B. and Störring, M. (2003) Facilitating the presence of users and 3D models by the augmented round table. In PRESENCE Conference, Aalborg, Denmark, October 2003.

Jackendoff, R. (1983) Semantics and Cognition. Cambridge, England: Cambridge University Press.

Lavergne, J. and Kimura, D. (1987) Hand movement asymmetry during speech: No effect of speaking topic. Neuropsychologia 25. pp. 689-693.

Lee, C.H., Ma, Y.P. and Jeng, T. (2003) A spatially-aware tangible user interface for computer-aided design. In Proceedings of the Conference on Human Factors in Computing Systems (CHI'03). pp 960-961.

Loomis, J.M. and Lederman, S.J. (1986) Tactual perception. In K.Boff, L. Kaufman and J. Tomas (eds.), Handbook Perception and Human Performance. New York: Wiley.

Magerkurth C. and Peter, T. (2002) Augmenting tabletop design for computer-supported cooperative work. In Workshop on Co-located Tabletop Collaboration: Technologies and Directions at CSCW'02, 2002.

Norman, D.A. (1988). The design of everyday things. New York: Basic Book.

Norman, D.A. (1991). Cognitive artefact. In J.M.Carroll (Ed.), Designing interaction. Cambridge, MA: Cambridge University Press.

Rizzo, A. and Marti, P. (1995) Distributed cognition and artefacts.

Seichter, H.and Kvan, T. (2004) Tangible interfaces in design computing, Virtual Environment 2.

Suwa, M., Purcell, T. and Gero, J. (1998) Macroscopic analysis of design processes based on a scheme for coding designer's cognitive actions. Design Studies, 19(4). pp 455-483.

Tang, J.C. (1991) Findings from observational studies of collaborative work. In International Journal of Man-Machine Studies, 34. pp 143-160.

Ullmer, B. and Ishii, H. (1997) The metaDESK: Models and prototypes for tangible user interfaces. In Proceedings of User Interface Software and Technology (UIST)' 97, 14-17 October, 1997. pp 14-21.

Underkoffler, J. and Ishii, H. (1999) Urp: a luminous-tangible workbench for urban planning and design. In Proceedings of the Conference on Human Factors in Computing Systems (CHI'99). pp 386-393.

Wang, Q., Li, C., Huang, X. and Tang, M. (2001) Tangible interface: integration of the real and virtual.

Wagner, S.M., Nusbaum, H. and Goldin-Meadow, S. (2004) Probing the mental representation of gesture: Is handwriting spatial? In the Journal of Memory and Language 50, pp 395-407. 